\title{
Changes in linoleic acid during follicular development and inhibition of spontaneous breakdown of germinal vesicles in cumulus-free bovine oocytes
}

\author{
S. T. Homa* and C. A. Brown \\ Department of Zoology, Arizona State University, Tempe, Arizona 85287, USA
}

\begin{abstract}
Summary. The fatty-acid composition of follicular fluid from small and large developing follicles was analysed and the effects of saturated and unsaturated fatty acids on spontaneous breakdown of germinal vesicles were investigated. Fatty acids were bound to bovine serum albumin and cultured with occytes at $100 \mu \mathrm{mol} / 1$. Linoleic acid (18:2) was the only fatty acid tested that significantly inhibited breakdown of germinal vesicles $(P<0.01)$. The effect was dose-dependent and was greatest at $50 \mu \mathrm{mol}$ fatty acid/1 (\% breakdown of control, $81 \cdot 1 \pm 6.8$ vs. $50 \mu$ mol linoleic acid/1, 35.4 $\pm 7 \cdot 3$; $P<0.02$ ). Linoleic acid was the major fatty acid, constituting about a third of the total fatty acid in the follicular fluid; followed by $18.9 \pm 1.0 \%$ and $16.9 \pm 1.3 \%$ oleic acid (18:1) in small and large follicles, respectively. Saturated fatty acids accounted for $<30 \%$ of the total fatty acid composition. There was a marked absence of tetraenoic acids in small and large follicles. Proportions of linoleic acid were significantly lower in follicular fluid from large follicles $(31.1 \pm 1.2 \%$ of total fatty acid) than from small follicles $(34.8 \pm 0.7 \%$ of total fatty acid $)(P<0.05)$ and there was a significant inverse correlation between follicle diameter and percentage of linoleic acid in the follicular fluid $(r=-0.6966 ; P<0.05)$. There was no significant alteration in any other fatty acid during follicular development. The results suggest that (i) a critical relative concentration of linoleic acid may be required for maintenance of meiotic arrest in bovine oocytes and (ii) the decline in the proportion of linoleic acid during follicular development may contribute to the relief of the inhibition of breakdown of germinal vesicles.
\end{abstract}

Keyrords: cow; fatty acid; meiosis; germinal vesicle; oocyte; follicle

\section{Introduction}

Oocytes develop in the primordial follicle of the embryo where they begin to undergo meiosis. In mammals, this process is arrested at the diplotene stage, or germinal vesicle stage, immediately before or shortly after birth (reviewed by McGaughey, 1983). Meiotic arrest persists until sexual maturity, when continued follicular growth and development is stimulated by follicle-stimulating hormone during each reproductive cycle. The preovulatory surge of luteinizing hormone results in the completion of follicular development accompanied by the resumption of meiosis (Ayalon et al., 1972). At this stage, the germinal vesicle breaks down, the chromatin condenses and the oocyte progresses to the metaphase of the second meiotic division.

Mammalian oocytes can spontaneously resume meiosis when removed from the follicular environment (reviewed by McGaughey, 1983); it was therefore suggested that components of follicular fluid regulate the maintenance of meiotic arrest (Chang, 1955). Several such factors have been reported to contribute to the maintenance of meiotic arrest, such as a peptide of low $M_{\mathrm{r}}$ in the 
follicular fluid (Gwatkin \& Anderson, 1976; Tsafriri et al., 1976), proportions of intrafollicular steroids (Rice \& McGaughey, 1981), and hypoxanthine and adenosine (Downs et al., 1985).

A previous study revealed that during the development of porcine follicles, actual concentrations of free fatty acid dramatically declined, accompanied by changes in the percentage distribution of fatty acids in the follicular fluid (Yao et al., 1980). The fatty acid composition of meiotically immature porcine oocytes reflects that of the follicular fiuid, being strikingly rich in polyunsaturated fatty acids (Homa et al., 1986). Data from a preliminary investigation suggested that linoleic acid (18:2), a polyunsaturated fatty acid, inhibits meiotic maturation in porcine oocytes (Homa, 1987). Since mammalian oocytes are bathed in follicular fluid and fatty acids play such a crucial role in cellular function (reviewed by Stubbs \& Smith, 1984), it was important to investigate further the relationship between fatty acids in follicular fluid and their influence in arresting meiosis in oocytes.

This study determined the fatty-acid composition of follicular fluid in small and large developing bovine follicles and investigated the effects of a range of saturated and unsaturated fatty acids on spontaneous breakdown of germinal vesicles in oocytes.

\section{Materials and Methods}

\section{Experiment 1: determination of fatty-acid composition of follicular fluid}

\section{Collection of follicular fluid for fatty-acid analysis}

Cow ovaries were purchased from a local abattoir. Follicle diameters were measured and follicular fluid was aspirated using an 18-gauge needle attached to a syringe. Care was taken to aspirate fluid only from follicles that appeared normal. Most antral follicles in mammalian ovaries are destined to undergo atresia (Himelstein-Braw et al., 1976; McGaughey et al., 1979; McGaughey, 1983); therefore, we cannot exclude the possibility that this process may have been initiated in a small percentage of our selected follicles, which may be reflected in the fatty-acid composition.

Fluid was pooled from several small follicles ( $1-3 \mathrm{~mm}$ diameter) of any one ovary to obtain a sufficient volume for analysis of fatty acids in any one sample. Large follicles ( $>7 \mathrm{~mm}$ diameter) contained sufficient fluid for us to carry out analysis without pooling. Five samples from five separate ovaries were prepared for each of the small- and largefollicle categories. The follicular fluid was centrifuged for $15 \mathrm{~min}$ at $13000 \mathrm{~g}$. Total lipids were extracted from $200-\mu \mathrm{l}$ aliquants of the supernatant.

\section{Fatty-acid analysis}

Total lipid extraction was achieved with the addition of $3.75 \mathrm{ml}$ chloroform/methanol $(1: 2 \mathrm{v} / \mathrm{v})$, followed by $1.25 \mathrm{ml}$ chloroform and $1.25 \mathrm{ml}$ water (Bligh \& Dyer, 1959). The organic phase was evaporated to dryness under a stream of $\mathrm{O}_{2}$-free $\mathrm{N}_{2}$, and saponified for $1 \mathrm{~h}$ at $80^{\circ} \mathrm{C}$ with $2 \mathrm{ml}$ of $33 \% \mathrm{KOH}$ in ethanol (6:94 v/v) containing $40 \mu \mathrm{g}$ hydroquinone $/ \mathrm{ml}$. After addition of $2 \mathrm{ml}$ water, the sample was washed twice with $3 \mathrm{ml}$ hexane. The fatty acids were extracted with two washes of $3 \mathrm{ml}$ hexane, after acidification with $1 \mathrm{~mol} \mathrm{HCl} / \mathrm{l}$, and evaporated to dryness under a stream of $\mathrm{O}_{2}$-free $\mathrm{N}_{2}$. Fatty-acid methyl esters were prepared by treatment with $3 \mathrm{ml}$ boron trifluoride in $14 \%$ methanol for $7 \mathrm{~min}$ at $80^{\circ} \mathrm{C}$ and extracted with three washes of $2 \mathrm{ml}$ hexane. The fatty-acid methyl esters were separated isothermally by gas-liquid chromatography (Hewlett-Packard model no. HP 5840A; Avondale, PA, USA) using a $30-\mathrm{m}$ Supelcowax column at $210^{\circ} \mathrm{C}$, with $\mathrm{N}_{2}$ as the carrier gas. The fatty-acid methyl esters were detected by flame ionization. The proportions of fatty acids were integrated automatically.

\section{Experiment 2: effects of fatty acids on breakdown of germinal vesicles}

\section{Collection of oocytes}

Oocytes were collected in chemically defined medium (2A-BMOC; McGaughey, 1977) using a procedure described by Homa (1988). Ovaries were macerated with double-edged razor blades, taking care to exclude corpora lutea and follicles $>5 \mathrm{~mm}$ in diameter. Large tissue debris was removed by passing the suspension through a $1 \mathrm{~mm}$ screen. Cumulus-enclosed oocytes were collected on a stainless steel screen with $106-\mu \mathrm{m}$ pores. The oocytes were denuded of adherent cumulus cells by vortexing with $1 \%(\mathrm{w} / \mathrm{v})$ sodium citrate in $1 \mathrm{~mol}$ EDTA/1 (pH 7.4) for $30 \mathrm{~s}$ and then resuspended in $2 \mathrm{~A}-\mathrm{BMOC}$ medium. The suspension was passed through a $106-\mu \mathrm{m}$ screen and the oocytes were washed on the screen surface with $2 \mathrm{~A}-\mathrm{BMOC}$ medium. The oocytes were then transferred to Petri dishes and selected for culture with the aid of a light microscope. Only oocytes exhibiting a uniform distribution of organelles within the cytoplasm were cultured. 


\section{Preparation of fatty acids bound to albumin}

Fatty acids (Sigma Chemical Co., St Louis, MO, USA, and NuCheck-Prep, Inc. Elysian, MN, USA) were bound to $10 \%(\mathrm{w} / \mathrm{v})$ essentially fatty-acid-free bovine serum albumin (BSA, Sigma) in 2A-BMOC medium using a modification of the method of Spector \& Hoak (1969). Fatty acids were dissolved in hexane $(2 \mathrm{mg} / \mathrm{ml})$ and added to celite ( $10 \mathrm{mg}$ fatty acid $/ 0.5 \mathrm{~g}$ celite). The hexane was evaporated under $\mathrm{O}_{2}$-free $\mathrm{N}_{2}$, and $10 \%$ BSA was added $(1 \mathrm{ml} 10 \%$ BSA $/ 2 \mathrm{mg}$ fatty acid). The albumin solution was left for $30 \mathrm{~min}$ at room temperature with occasional agitation and then decanted and centrifuged for $15 \mathrm{~min}$ at $13000 \mathrm{~g}$. The solutions were sterilized by passing them through a $22-\mu \mathrm{m}$ millipore filter (Millipore Corp., Bedford, MA, USA). The amount of fatty acid bound to albumin was determined by gas liquid chromatography. Concentrations were adjusted with $10 \%$ BSA in 2A-BMOC medium.

\section{Oocyte culture}

(a) Effect of $C_{16}$ and $C_{18}$ fatty acids on breakdown of germinal vesicles. Groups of oocytes (6-10/group representing one replicate) were incubated under sterile conditions in $190 \mu \mathrm{l} 2 \mathrm{~A}-\mathrm{BMOC}$ medium with $10 \mu \mathrm{l}$ palmitic (16:0), palmitoleic (16:1), stearic (18:0), oleic (18:1), linoleic (18:2) or $\alpha$-linolenic (18:3) acid bound to albumin to give $100 \mu \mathrm{mol}$ fatty acid/l. This concentration was predicted to be well within the physiological range compared with values calculated for fatty-acid concentrations in porcine follicular fluid (Yao et al., 1980). The BSA concentration was $0.5 \%(\mathrm{w} / \mathrm{v})$; in control experiments, $0.5 \%$ BSA was used alone. Generally, two or three replicates were carried out for each treatment, and the experiments were carried out three times. Incubations were carried out for $24 \mathrm{~h}$ in Lab-Tek chamber slides (Nunc, Inc., Naperville, IL, USA) at $38^{\circ} \mathrm{C}$ under $5 \% \mathrm{CO}_{2}+5 \% \mathrm{O}_{2}+90 \% \mathrm{~N}_{2}$ in a humidified atmosphere.

(b) Effect of $C_{20}$ and $C_{22}$ fatty acids on breakdown of germinal vesicles. Groups of oocytes were cultured with $100 \mu \mathrm{mol}$ arachidonic (20:4), eicosapentaenoic (20:5) or adrenic (22:4) acid/l under the conditions described for Expt 2a.

(c) Effect of concentration of linoleic acid on breakdown of germinal vesicles. The dose response was investigated by culturing groups of oocytes with $0-200 \mu \mathrm{mol}$ linoleic acid $/ \mathrm{l}$. The BSA concentration was maintained at $0.5 \%$. Culture conditions were as described for Expt $2 \mathrm{a}$ and $2 \mathrm{~b}$.

\section{Determination of stage of meiosis}

Oocytes were placed in $1 \%$ sodium citrate and were subsequently fixed in ethanol:acetic acid ( $3: 1 \mathrm{v} / \mathrm{v})$, allowed to air-dry and stained in Wright's stain in $2.9 \%(\mathrm{w} / \mathrm{v})$ glycerol in methanol (Rice \& McGaughey, 1981). Oocyte maturation was scored cytogenetically: at the germinal vesicle stage, chromatin was classified as either normal (fibrous or diffuse) or degenerate, as described by McGaughey et al. (1979) and Homa (1988). Oocytes which had progressed to or beyond diakinesis were considered to have undergone breakdown of germinal vesicles and to be maturing (McGaughey et al., 1979).

\section{Statistical analysis}

Statistical analyses were carried out using Student's $t$ test. Values of $P<0.05$ were considered significant.

\section{Results}

\section{Experiment 1: fatty-acid composition of follicular fluid}

Saturated fatty acids accounted for $<30 \%$ of the total fatty acids in both small and large follicles (Table 1); polyunsaturated fatty acids constituted the major components $(48.0 \%$ in small follicles, $46.7 \%$ in large follicles). The primary constituent in bovine follicular fluid was the dienoic fatty acid linoleic acid (18:2), contributing about a third of the total fatty acid composition, almost twice as much as the secondary fatty acid component, the monoenoic oleic acid (18:1) (Table 1). The saturated fatty acids palmitic (16:0) and stearic (18:0) acids were present in similar proportions, representing 13.3 and $11.0 \%$, respectively, in fluid from small follicles, and 14.8 and $12.3 \%$, respectively, in fluid from large follicles. There was a notable absence of arachidonic acid (20:4) in fluid from small and large follicles, and adrenic acid (22:4) was negligible. The fatty-acid profile of fluid from large follicles appeared to contain greater proportions of palmitic, palmitoleic (16:1), stearic and linolenic (18:3) acids than fluid from small follicles, but these differences were not statistically significant; the lower proportion of oleic acid was not significant; but the percentage of linoleic acid was significantly lower in fluid from large follicles $(31 \cdot 1 \%)$ than in that of small follicles $(34.8 \% ; P<0.05 ;$ Table 1$)$. When the proportion of linoleic acid, expressed as a percentage of the total fatty acid in follicular fluid, was plotted against the average follicle diameter (Fig. 1), a significant inverse correlation was revealed $(P<0 \cdot 05)$. 
Table 1. Fatty acid composition of bovine follicular fluid

\begin{tabular}{lcc}
\hline Fatty acid & $\begin{array}{c}\text { Small follicles } \\
(1-3 \mathrm{~mm} \text { diameter })\end{array}$ & $\begin{array}{c}\text { Large follicles } \\
(7-13 \mathrm{~mm} \text { diameter })\end{array}$ \\
\hline Palmitic (16:0) & $13 \cdot 3 \pm 0 \cdot 7$ & $14 \cdot 8 \pm 1 \cdot 4$ \\
Palmitoleic (16:1) & $5 \cdot 0 \pm 0 \cdot 5$ & $6 \cdot 4 \pm 1 \cdot 3$ \\
Stearic (18:0) & $11 \cdot 0 \pm 1 \cdot 1$ & $12 \cdot 3 \pm 0 \cdot 9$ \\
Oleic (18:1) & $18 \cdot 9 \pm 1 \cdot 0$ & $16 \cdot 9 \pm 1 \cdot 3$ \\
Linoleic (18:2) & $34 \cdot 8 \pm 0 \cdot 7^{*}$ & $31 \cdot 1 \pm 1 \cdot 2^{*}$ \\
$\gamma$-Linolenic (18:3 n-6) & $1 \cdot 1 \pm 0 \cdot 3$ & $0 \cdot 6 \pm 0 \cdot 4$ \\
$\alpha$-Linolenic (18:3 n-3) & $6 \cdot 1 \pm 0 \cdot 5$ & $8 \cdot 1 \pm 1 \cdot 4$ \\
Arachidic (20:0) & $1 \cdot 3 \pm 0 \cdot 4$ & $0 \cdot 6 \pm 0 \cdot 4$ \\
Eicosenoic (20:1) & $1 \cdot 3 \pm 0 \cdot 4$ & $0 \cdot 8 \pm 0 \cdot 4$ \\
Eicosadienoic (20:2) & $1 \cdot 0 \pm 0 \cdot 4$ & $0 \cdot 6 \pm 0 \cdot 3$ \\
Eicosatrienoic (20:3) & $3 \cdot 2 \pm 0 \cdot 5$ & $3 \cdot 0 \pm 0 \cdot 4$ \\
Eicosapentaenoic (20:5) & $1 \cdot 0 \pm 0 \cdot 5$ & $1 \cdot 7 \pm 0 \cdot 3$ \\
Heneicosanoic (21:0) & $1 \cdot 2 \pm 0 \cdot 4$ & $1 \cdot 6 \pm 0 \cdot 2$ \\
Docosapentaenoic (22:5) & $0 \cdot 8 \pm 0 \cdot 3$ & $1 \cdot 6 \pm 0 \cdot 3$ \\
\hline
\end{tabular}

Values are the means \pm s.e.m. of five separate sample determinations, and are expressed as a percentage of the total fatty acid composition. Only fatty acids constituting $>1 \%$ of the total in either follicle category were included.

*Value of linoleic acid in small follicles significantly different from value of linoleic acid in large follicles $(P<0.05)$.

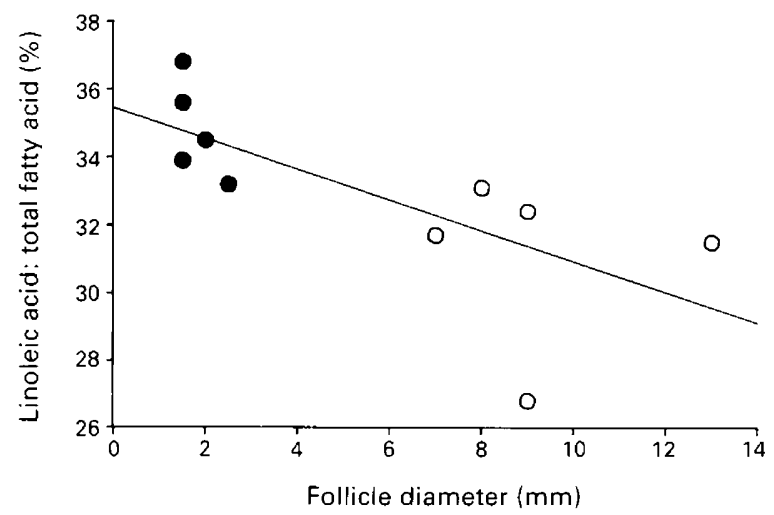

Fig. 1. Correlation between the proportion of linoleic acid in the follicular fluid and diameter of bovine follicle; $(\Theta)$ averaged for pooled samples, $1-3 \mathrm{~mm}$ diameter; $(O)$ individual diameters for unpooled samples; $r=-0.6966, P<0.05$.

\section{Experiment 2: effects of fatty acids on breakdown of germinal vesicles}

In the absence of fatty acid, 85.6-94.6\% of oocytes resumed meiosis (controls for Expt $2 \mathrm{a}$ and 2 b, respectively; Table 2). Linoleic acid (18:2) significantly inhibited resumption of meiosis; only $48.6 \%$ of oocytes demonstrated breakdown of germinal vesicles in the presence of the fatty acid $(P<0.01)$ (Table 2). However, this fatty acid did not affect the ability of oocytes that did undergo breakdown in its presence to progress to anaphase I or beyond (Table 2; $47.8 \%$ of total maturing oocytes in the absence of fatty acid vs. $51.4 \%$ of total maturing oocytes in the presence of linoleic acid). None of the other fatty acids tested had any significant effect on the resumption or progression of meiosis. The proportion of oocytes which degenerated during culture in the presence of any fatty acid was not significantly different from that in the absence of fatty acid (Table 3 ). 
Table 2. Effects of fatty acids on breakdown of germinal vesicles and meiotic progression in bovine oocytes

\begin{tabular}{|c|c|c|c|}
\hline Fatty acid & $\begin{array}{l}\text { Number of } \\
\text { replicates }\end{array}$ & $\begin{array}{c}\text { Breakdown of } \\
\text { germinal vesicles } \\
(\%)^{*}\end{array}$ & $\begin{array}{c}\text { Anaphase I to } \\
\text { metaphase II } \\
(\%) \dagger\end{array}$ \\
\hline \multicolumn{4}{|l|}{ Experiment 2a } \\
\hline None (control) & 8 & $85 \cdot 6 \pm 5 \cdot 3^{a}$ & $47 \cdot 8 \pm 10 \cdot 4$ \\
\hline Palmitic $(16: 0)$ & 2 & $70 \cdot 1(75 \cdot 0,66 \cdot 7)$ & $50 \cdot 0(100,0)$ \\
\hline Palmitoleic (16:1) & 8 & $63 \cdot 6 \pm 12 \cdot 8$ & $65 \cdot 3 \pm 11 \cdot 1$ \\
\hline Stearic $(18: 0)$ & 9 & $61.7 \pm 9.4$ & $41.5 \pm 12 \cdot 7$ \\
\hline Oleic $(18: 1)$ & 8 & $70 \cdot 2 \pm 5 \cdot 5$ & $66.4 \pm 7.9$ \\
\hline Linoleic $(18: 2)$ & 8 & $48 \cdot 6 \pm 10 \cdot 3^{a}$ & $51.4 \pm 15 \cdot 3$ \\
\hline$\alpha$-Linolenic (18:3) & 8 & $74.6 \pm 7 \cdot 4$ & $59 \cdot 1 \pm 14 \cdot 2$ \\
\hline \multicolumn{4}{|l|}{ Experiment 2b } \\
\hline None (control) & 8 & $94 \cdot 6 \pm 4 \cdot 5$ & $83 \cdot 3 \pm 6.4$ \\
\hline Arachidonic (20:4) & 6 & $84.9 \pm 6.0$ & $74.2 \pm 9.8$ \\
\hline Eicosapentaenoic $(20: 5)$ & 4 & $92 \cdot 2 \pm 5 \cdot 3$ & $85.9 \pm 5.8$ \\
\hline Adrenic $(22: 4)$ & 5 & $82.7 \pm 7.8$ & $61 \cdot 4 \pm 12 \cdot 5$ \\
\hline
\end{tabular}

Oocytes were cultured with, or without, $100 \mu \mathrm{mol}$ fatty acid/1 bound to bovine serum albumin.

*Percentage of total non-degenerate oocytes \pm s.e.m.

$\neq$ Percentage of total oocytes undergoing breakdown \pm s.e.m. for $\%$ GVBD in the presence of linoleic acid vs. control, $P<0 \cdot 01$.

apercentage breakdown of germinal vesicles in the presence of linoleic acid vs. control, $P<0.01$.

Table 3. Incidence of degenerate bovine oocytes in the presence of fatty acids

\begin{tabular}{lc}
\hline Fatty acids & $\begin{array}{c}\text { Degenerate oocytes } \\
(\%)^{*}\end{array}$ \\
\hline Experiment 2a & $20 \cdot 7 \pm 4 \cdot 3$ \\
None (control) & $20 \cdot 0(0.40 \cdot 0)$ \\
Palmitic (16:0) & $28 \cdot 6 \pm 7 \cdot 2$ \\
Palmitoleic (16:1) & $26 \cdot 1 \pm 4 \cdot 4$ \\
Stearic (18:0) & $27 \cdot 4 \pm 2 \cdot 2$ \\
Oleic (18:1) & $25 \cdot 3 \pm 6 \cdot 4$ \\
Linoleic (18:2) & $25 \cdot 6 \pm 3 \cdot 9$ \\
$\alpha-$ Linolenic (18:3) & \\
Experiment 2b & $12 \cdot 9 \pm 4 \cdot 9$ \\
None (control) & $16 \cdot 3 \pm 8 \cdot 5$ \\
Arachidonic (20:4) & $18 \cdot 5 \pm 5 \cdot 0$ \\
Eicosapentaenoic (20:5) & $18 \cdot 7 \pm 6 \cdot 0$ \\
Adrenic (22:4) & \\
\hline *Values are means \pm s.e.m. of all oocytes cultured in \\
$\quad$ Expts 2a and 2b.
\end{tabular}

The effect of linoleic acid was dose-dependent (Fig. 2), reaching maximum inhibition of breakdown of germinal vesicles at $50 \mu \mathrm{mol} / 1$ (\% breakdown of control, $81 \cdot 1 \pm 1 \cdot 1 \mathrm{vs}$. $50 \mu \mathrm{mol}$ linoleic acid/1, 35.4 $\pm 7 \cdot 3 ; P<0.02$ ). The proportion of oocytes prevented from undergoing breakdown of germinal vesicles did not increase significantly at higher doses. Incubation of bovine oocytes with linoleic acid bound to BSA revealed that the concentrations of fatty acid used in these experiments were not toxic, as the proportion of oocytes that were degenerate was not significantly different in the control and the groups treated with fatty acid (Table 2). 


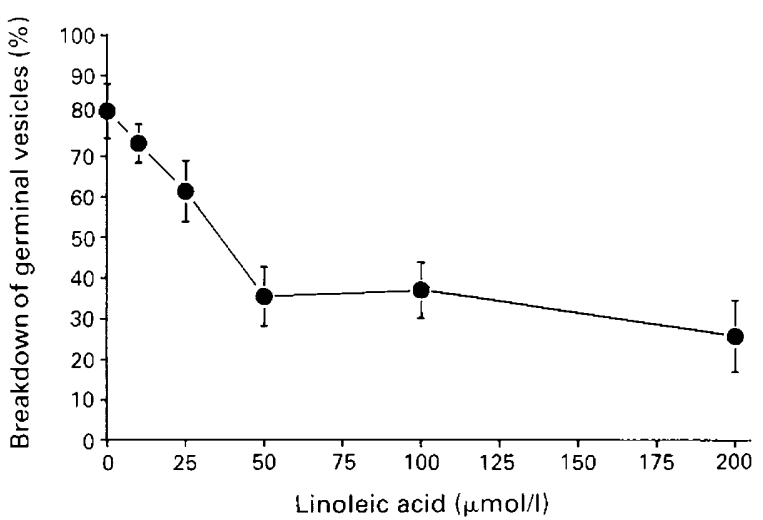

Fig. 2. Response of breakdown of germinal vesicles in bovine oocytes to concentration of linoleic acid. Values are means \pm s.e.m. of percentage breakdown in the presence of $50 \mu \mathrm{mol}$ linoleic acid $/ 1$ vs. control, $P<0.02$.

\section{Discussion}

The observation that linoleic acid significantly inhibited breakdown of germinal vesicles is intriguing. In vivo, oocytes in small follicles are developmentally immature and meiosis is arrested. It is only after the preovulatory follicle has grown to a large stage that the inhibitory influence upon resumption of meiosis in oocytes is released, under the influence of luteinizing hormone (see McGaughey, 1983). The effect of linoleic acid may be physiologically relevant: first, this fatty acid is a natural constituent of follicular fluid in which the oocyte is bathed and, secondly, the actual concentration of free fatty acid in porcine follicular fluid is $\sim 1 \mathrm{mmol} / 1$ (Yao et al., 1980) and we have demonstrated inhibition of breakdown of germinal vesicles in bovine oocytes by only $50 \mu \mathrm{mol}$ linoleic acid/l.

The results from the present study indicate that the fatty-acid environment of immature bovine oocytes in small follicles is more conducive to maintaining meiotic arrest than that of developing follicles, since the proportion of linoleic acid is smaller in the latter. This suggests that a critical relative concentration of linoleic acid may be necessary for the maintenance of meiotic arrest in bovine oocytes. The actual concentrations of fatty acids were not measured in this study, but it is interesting that the concentration of total fatty acid declines significantly in porcine follicular fluid as the follicle increases in size (Yao et al., 1980).

Cyclic adenosine monophosphate (cAMP) plays a significant role in the maintenance of oocyte meiotic arrest in several mammalian species (mouse, Cho et al., 1974; rat, Magnusson \& Hillensjö, 1977; pig, Rice \& McGaughey, 1981, Racowsky, 1985a; cow, Ball et al., 1983; hamster, Racowsky, 1985b; cow, Homa, 1988). Incorporation of unsaturated fatty acids into membrane phospholipids increases basal and stimulated activity of adenylate cyclase (Engelhard et al., 1978; Colard et al., 1980; Poon et al., 1981; Chambaz et al., 1983). The stimulatory effect is specific for incorporation of linoleic acid (Colard et al., 1980; Chambaz et al., 1983). Evidence supports the existence of adenylate cyclase in the mammalian oocyte plasma membrane (Racowsky, 1985a, b), and it is therefore tempting to speculate that the inhibitory effect of linoleic acid on spontaneous breakdown of germinal vesicles in bovine oocytes may be due, in part, to its specific stimulatory effect on activity of adenylate cyclase.

Linoleic acid stimulates protein kinase C (Murakami et al., 1986; Dell \& Severson, 1989), which plays a significant role in cell growth and differentiation (Nishizuka, 1988) and may be important in regulating meiotic arrest. Tumour-promoting phorbol esters, which permanently activate protein kinase C, inhibit the resumption of meiosis in murine oocytes (Urner \& Schorderet-Slatkine, 1984; 
Bornslaeger et al., 1986) and promote the inhibitory effects of a cAMP phosphodiesterase inhibitor on breakdown of germinal vesicles in bovine oocytes (Homa, 1991). Thus, the effects of linoleic acid may result from a direct activation of protein kinase $\mathrm{C}$, thereby mimicking the effect of tumourpromoting phorbol esters on oocyte maturation.

Alternatively, linoleic acid may exert its effect through prostaglandins and/or leukotrienes, which have potent effects as local hormones (Smith, 1989) and have been implicated in ovarian function (reviewed by Armstrong, 1981). Lipoxygenase activity has been detected in oocytes of several invertebrates and lower vertebrates (sea-urchin, Perry \& Epel, 1985; starfish, Meijer et al., 1986; frog, Hawkins \& Brash, 1989). Although arachidonic acid (20:4), the primary substrate for both lipoxygenase and cyclooxygenase (Smith, 1989), does not occur in bovine follicular fluid, linoleic acid can serve as an alternative precursor (Claeys et al., 1985; Hawkins \& Brash, 1989).

Irrespective of the mechanisms involved in inhibition by linoleic acid of the resumption of meiosis, dietary alterations may modify the fatty-acid composition of follicular fluid, since it is partly derived from serum (Yao et al., 1980). As oocytes are bathed in follicular fluid, a high intake of polyunsaturated fatty acids in the diet may potentially influence the ability of oocytes to mature in vivo. The fatty-acid patterns of individual lipids of meiotically immature porcine oocytes (Homa et al., 1986) are similar to that of porcine follicular fluid, suggesting a direct relationship between concentrations of fatty acids in oocytes and follicular fluid.

The fatty-acid profiles of bovine follicular fluid differ from those reported for total lipid extracts of porcine follicular fluid (Yao et al., 1980). The most prominent fatty acid in porcine follicular fluid was oleic acid (18:1), followed by palmitic acid (16:0), compared with linoleic acid (18:2), followed by oleic acid in bovine follicular fluid. Porcine follicles contained $\sim 8 \%$ arachidonic acid (20:4) and measurable amounts of adrenic acid (22:4), but there was a marked absence of these tetraenoic acids in bovine follicular fluid. During development of bovine follicles, only the proportion of linoleic acid changed significantly; whereas, during development of porcine follicles, proportions of polyunsaturated fatty acids and stearic acid (18:0) increased, and palmitic and oleic acids decreased. These results suggest that the fatty-acid distribution in mammalian follicular fluid is species-specific.

The authors would like to thank R. Russell for excellent technical assistance, and E. Williams for carrying out the gas-liquid chromatography. This study was supported by grant no. HD 20884 from the National Institutes of Health to S. T. Homa.

\section{References}

Armstrong, D.T. (1981) Prostaglandins and follicular functions. J. Reprod. Fert. 62, 283-291.

Ayalon, D., Tsafriri, A., Lindner, H.R., Cordova, T. \& Harell, A. (1972) Serum gonadotrophin levels in proestrous rats in relation to the resumption of meiosis by oocytes. J. Reprod. Fert. 31, 37-58.

Ball, G.D., Lenz, R.W., Ax, R.L. \& First, N.L. (1983) Effects of cAMP on bovine oocyte maturation and cumulus expansion. In Factors Regulating Ovarian Function, pp. 61-64. Eds G. S. Greenwald \& P. F. Terranova. Raven Press, New York.

Bligh, E.G. \& Dyer, W.J. (1959) A rapid method of total lipid extraction and purification. Can. J. Biochem. Phisiol. 37, 911-917.

Bornslaeger, E.A., Poueymirou, W.T., Mattei, P. \& Schultz, R.M. (1986) Effects of protein kinase C activators on germinal vesicle breakdown and polar body emission of mouse oocytes. Exp. Cell Res. 165, 507517.

Chambaz, J., Pepin, D., Robert, A., Wolf, C. \& Bereziat,
G. (1983) Protein-stimulated enrichment of human platelet membranes in linoleyl-phosphatidylcholines. Effect upon adenylate cyclase and fluidity. Biochim. biophys. Acta 727, 313-326.

Chang, M.C. (1955) The maturation of rabbit oocytes in culture and their maturation, activation, fertilization, and subsequent development in the fallopian tubes. $J$. exp. Zool. 128, $378-405$.

Cho, W.K., Stern, S. \& Biggers, J.D. (1974) Inhibitory effect of dibutyryl cAMP on mouse oocyte maturation in vitro. J. exp. Zool. 187, 383-386.

Claeys, M., Kivits, G.A.A., Christ-Hazelhof, E. \& Nugteren, D.H. (1985) Metabolic profiles of linoleic acid in porcine leukocytes through the lipoxygenase pathway. Biochim. biophys. Acta 837, 35-51.

Colard, O., Kervabon, A. \& Roy, C. (1980) Effects on adenylate cyclase activities of unsaturated fatty acid incorporation into rat liver plasma membrane phospholipids. Specific modulation by linoleate. Biochem. biophys. Res. Commun. 95, 97-102.

Downloaded from Bioscientifica.com at 04/26/2023 10:33:36AM 
Dell, K.R. \& Severson, D.L. (1989) Effect of cisunsaturated fatty acids on aortic protein kinase $\mathrm{C}$ activity. Biochem. J. 258, 171-175.

Downs, S.M., Coleman, D.L., Ward-Bailey, P.F. \& Eppig, J. (1985) Hypoxanthine is the principal inhibitor of murine oocyte maturation in a low-molecularweight fraction of porcine follicular fluid. Proc. natl Acad. Sci. USA 82, 454-458.

Engelhard, V.H., Glaser, M. \& Storm, D.R. (1978) Effect of membrane phospholipid compositional changes on adenylate cyclase in LM cells. Biochemistry, $N Y$ $17,319[-3200$.

Gwatkin, R.B. \& Anderson, O.F. (1976) Hamster oocyte maturation in vitro: Inhibition by follicular components. Life Sci. 19, 527-536.

Hawkins, D.J. \& Brash, A.R. (1989) Lipoxygenase metabolism of polyunsaturated fatty acids in oocytes of the frog Xenopus laevis. Arch. Biochem. Biophys. 268, 447-455

Himelstein-Braw, R., Byskov, A.G., Peters, H. \& Faber, M. (1976) Follicular atresia in the infant human ovary. J. Reprod. Fert. 46, 55-59.

Homa, S.T. (1987) Effects of fatty acids on oocyte maturation. Fed. Proc. 46, 2126.

Homa, S.T. (1988) Effects of cyclic AMP on the spontaneous meiotic maturation of cumulus-free bovine oocytes cultured in chemically defined medium. $J$. exp. Zool. 248, 222 231.

Homa, S.T. (1991) Neomycin, an inhibitor of phosphoinositide hydrolysis, inhibits the resumption of bovine oocyte spontaneous meiotic maturation. $J$. exp. Zool. 258, 95-103.

Homa, S.T., Racowsky, C. \& McGaughey, R.W. (1986) Lipid analysis of immature pig oocytes. J. Reprod. Fert. 77, 425-434.

Magnusson, C. \& Hillensjö, T. (1977) Inhibition of maturation and metabolism in rat oocytes by cyclic AMP J. exp. Zool. 201, 139147.

McGaughey, R.W. (1977) The maturation of porcine oocytes in minimal, defined culture media with varied macromolecular supplements and varied osmolarity. Exp. Cell Res. 109, 25-30.

McGaughey, R.W. (1983) Regulation of oocyte maturation. Oxford Rev. Reprod. Biol. 5, 106-130.

McGaughey, R.W., Montgomery, D.H. \& Richter, J.D. (1979) Germinal vesicle configurations and patterns of polypeptide synthesis of porcine oocytes from antral follicles of different size, as related to their competency for spontaneous maturation. J. exp. Zool. 209, 239-254.

Meijer, L., Maclouf, J. \& Bryant, R.W. (1986) Arachidonic acid metabolism in starfish oocytes. Devl Biol. 114, 22-23.
Murakami, K., Chan, S.Y. \& Routtenberg, A. (1986) Protein kinase $C$ activation by cis-fatty acid in the absence of $\mathrm{Ca}^{2+}$ and phospholipids. J. biol. Chem. 261, 15424-15429.

Nishizuka, Y. (1988) The molecular heterogeneity of protein kinase $\mathrm{C}$ and its implications for cellular regulation. Nature, Lond. 334, 661-665.

Perry, G. \& Epel, D. (1985) Characterization of a $\mathrm{Ca}^{2+}$ stimulated lipid peroxidising system in the sea urchin egg. Devl Biol. 107, 47-57.

Poon, R., Richards, J.M. \& Clark, W.R. (1981) The relationship between plasma membrane lipid composition and physical-chemical properties. II. Effect of phospholipid fatty acid modulation on plasma membrane physical properties and enzymatic activities. Biochim. biophys. Acta 649, 58-66.

Racowsky, C. (1985a) Effect of forskolin on maintenance of meiotic arrest and stimulation of cumulus expansion, progesterone and cyclic AMP production by pig oocyte-cumulus complexes. J. Reprod. Fert. 74, 9-2I.

Racowsky, C. (1985b) Effect of forskolin on the spontaneous maturation and cyclic AMP content of hamster oocyte cumulus complexes. J. exp. Zool. 234, 87-96.

Rice, C. \& McGaughey, R.W. (1981) Effect of testosterone and dibutyryl cAMP on the spontaneous maturation of pig oocytes. J. Reprod. Fert. 62, $245-256$.

Smith, W.L. (1989) The eicosanoids and their biochemical mechanisms of action. Biochem. J. 259, 315-324.

Spector, A.A. \& Hoak, J.C. (1969) An improved method for the addition of long-chain free fatty acid to protein solutions. Analyt. Biochem. 32, 297-302.

Stubbs, C.D. \& Smith, A.D. (1984) The modification of mammalian membrane polyunsaturated fatty acid composition in relation to membrane fluidity and function. Biochim. biophys. Acta 779, 89-137.

Tsafriri, A., Pomerantz, S.H. \& Channing, C.P. (1976) Inhibition of oocyte maturation by porcine follicular fluid: partial characterization of the inhibitor. Biol. Reprod. 14, 511-516.

Urner, F. \& Schorderet-Slatkine, S. (1984) Inhibition of denuded mouse oocyte meiotic maturation by tumor-promoting phorbol esters and its reversal by retinoids. Exp. Cell Res. 154, 600-605.

Yao, J.K., Ryan, R.J. \& Dyck, P.J. (1980) The porcine ovarian follicle. VI. Comparison of fatty acid composition of serum and follicular fluid at different developmental stages. Biol. Reprod. 22, 141-147.

Received 29 November 1990 